\title{
Compensated Compactness in Banach Spaces and Weak Rigidity of Isometric Immersions of Manifolds
}

\author{
Gui-Qiang G. Chen and Siran Li \\ To Helge Holden on the occasion of his 60 th birthday \\ with friendship and affection
}

\begin{abstract}
We present a compensated compactness theorem in Banach spaces established recently, whose formulation is originally motivated by the weak rigidity problem for isometric immersions of manifolds with lower regularity. As a corollary, a geometrically intrinsic div-curl lemma for tensor fields on Riemannian manifolds is obtained. Then we show how this intrinsic div-curl lemma can be employed to establish the global weak rigidity of the Gauss-Codazzi-Ricci equations, the Cartan formalism, and the corresponding isometric immersions of Riemannian submanifolds.
\end{abstract}

2010 Mathematics Subject Classification. Primary: 53C24, 53C42, 53C21, 53C45, 57R42, 35M30, 35B35, 58A15, 58J10; Secondary: 57R40, 58A14, 58A17, 58A05, 58K30, 58Z05.

Keywords. Compensated compactness, weak rigidity, global, intrinsic, Gauss-CodazziRicci equations, Riemannian manifolds, isometric immersions, isometric embeddings, lower regularity, weak convergence, approximate solutions, div-curl lemma, Riemann curvature.

\section{Introduction}

In this paper we discuss a unified approach developed recently in [1] towards establishing more general and intrinsic compensated compactness theorems for nonlinear analysis and nonlinear partial differential equations (PDEs), with applications to the weak rigidity of isometric immersions of Riemannian manifolds into Euclidean spaces with lower regularity.

Compensated compactness has played an important role in the study of nonlinear PDEs arising from fluid mechanics, calculus of variations, and nonlinear elasticity; cf. [2, 16, 20, 21, 24, 39, 50, 51] and the references cited therein. The div-curl lemma introduced by Murat and Tartar [39, 50, is the cornerstone of the theory, which reads in the simplest and original form as follows:

Lemma 1.1 (The div-curl lemma by Murat-Tartar [39, 50]). Let $\left\{v^{\epsilon}\right\},\left\{w^{\epsilon}\right\} \subset$ $L_{\text {loc }}^{2}\left(\mathbb{R}^{3} ; \mathbb{R}^{3}\right)$ be two sequences of vector fields on $\mathbb{R}^{3}$ such that

$$
v^{\epsilon} \rightarrow \bar{v}, \quad w^{\epsilon} \rightarrow \bar{w} \quad \text { weakly in } L_{l o c}^{2} .
$$


Assume that $\left\{\operatorname{div} v^{\epsilon}\right\}$ is pre-compact in $H_{\text {loc }}^{-1}\left(\mathbb{R}^{3} ; \mathbb{R}\right)$ and $\left\{\operatorname{curl} w^{\epsilon}\right\}$ is pre-compact in $H_{\text {loc }}^{-1}\left(\mathbb{R}^{3} ; \mathbb{R}^{3}\right)$. Then

$$
v^{\epsilon} \cdot w^{\epsilon} \rightarrow \bar{v} \cdot \bar{w} \quad \text { in the sense of distributions. }
$$

Two distinctive approaches have been developed in the literature to prove Lemma 1.1? One is via harmonic analysis, and the other is based on the Hodge (de Rham) decomposition theorem; see [16, 23, 33, 40, 45] and the references cited therein. Both approaches depend crucially on the geometry of the Euclidean spaces or Riemannian manifolds; see $\S 2$ for a detailed exposition. See also Whitney [54] (Chapter IX, Theorem 17A) for an early version of the div-curl type lemma in the language of his geometric integration theory.

One of our crucial observations in [11] is that the div-curl lemma can be reformulated via a functional-analytic approach in generic Banach spaces with two general bounded linear operators in place of div and curl. Indeed, all the necessary properties we need for div and curl in order to conclude the lemma can be extracted as two simple, abstract conditions: One is algebraic, and the other is analytic. Both conditions can be naturally formulated in terms of operator algebras on Banach spaces. This leads to the generalization of the existing versions of the div-curl lemma, and provides the third approach to the theory of compensated compactness. In addition, combining the functionalanalytic compensated compactness theorem together with the ellipticity of the Laplace-Beltrami operator, we are ready to obtain a geometrically intrinsic div-curl lemma on Riemannian manifolds. Throughout this paper, the term "intrinsic" means "independent of local coordinates" on Riemannian manifolds.

As an application of the new div-curl lemma, we analyze the weak rigidity of isometric immersions of Riemannian manifolds into Euclidean spaces. The problem of isometric immersions/embeddings has been of considerable interest in the development of differential geometry, which has also led to the important developments of new ideas and methods in nonlinear analysis and PDEs ( $c f$. [30, 42, 43, 55]). Moreover, it is well-known in differential geometry that the Gauss-Codazzi-Ricci (GCR) equations are the compatibility conditions for the existence of isometric immersions (see [19, 46]). The GCR equations can be viewed as a first-order nonlinear system of geometric PDEs for the second fundamental forms and normal connections. However, in general, the GCR equations are of no type, neither purely hyperbolic nor purely elliptic.

The weak rigidity problem for isometric immersions can be formulated as follows: Given a sequence of isometric immersions of an $n$-dimensional manifold with a $W_{l o c}^{1, p}$ metric for $p>n$, whose second fundamental forms and normal connections are uniformly bounded in $L_{\text {loc }}^{p}$, whether its weak limit is still an isometric immersion with the same $W_{\text {loc }}^{1, p}$ metric. This rigidity problem has its motivation from both geometric analysis and nonlinear elasticity: The existence of isometric immersions of Riemannian manifolds with lower regularity corresponds naturally to the realization of elastic bodies with lower regularity 
in the physical space. See Ciarlet-Gratie-Mardare [15], Mardare [36], Szopos [49, and the references cited therein.

In [11, we have proved that the solvability of the GCR equations in $W^{1, p}$ is equivalent to the existence of $W^{2, p}$ isometric immersions on Riemannian manifolds. This is done by employing the Cartan formalism, also known as the method of moving frames. We have shown that both the GCR equations and isometric immersions are equivalent to the structural equations of the Cartan formalism. Then, by exploiting the div-curl structure of the GCR equations and the Cartan formalism, we have deduced the global weak rigidity of these geometric PDEs, independent of local coordinates on Riemannian manifolds. Now, in view of the equivalence theorem established above, the weak rigidity of isometric immersions is readily concluded.

The rest of this paper is organized as follows: In $\S 2$, we first formulate the functional-analytic compensated compactness theorem in Banach spaces and give an outline of its proof, and then deduce a geometrically intrinsic div-curl lemma on Romannian manifolds as its corollary. Two generalizations of the latter result are also discussed. In $\S 3$, we collect some background on differential geometry pertaining to the GCR equations and the Cartan formalism. Finally, in $\S 4$, we show the weak rigidity of isometric immersions, together with the weak rigidity of the GCR equations and the Cartan formalism.

\section{A Compensated Compactness Theorem in Banach Spaces}

In this section we first discuss a functional-analytic compensated compactness theorem. As its consequence, we deduce a geometrically intrinsic div-curl lemma on Riemannian manifolds.

To establish the original div-curl lemma, Lemma 1.1, as well as its various generalizations (see [16, 23, 33, 40, 45] and the references cited therein), the following distinctive approaches have been adopted:

The first approach, developed by Murat and Tartar in [39, 50, is based on harmonic analysis. It is observed that the first-order differential constraints, namely the pre-compactness of $\left\{\operatorname{div} v^{\epsilon}\right\}$ and $\left\{\operatorname{curl} w^{\epsilon}\right\}$ in $H^{-1}$, lead to the decay properties of $\left\{v^{\epsilon} \cdot w^{\epsilon}\right\}$ in the high Fourier frequency region. Coifman-LionsMeyer-Semmes in [16] extended this lemma by combining the exploitation of this observation with further techniques in harmonic analysis, including Hardy spaces, and commutator estimates of BMO functions and Riesz transforms.

The second approach is based on the Hodge decomposition. Robbin-RogersTemple in [45] observed that, by writing

$$
v^{\epsilon}=\Delta \Delta^{-1} v^{\epsilon}=(\operatorname{grad} \circ \operatorname{div}-\operatorname{curl} \circ \operatorname{curl}) \Delta^{-1} v^{\epsilon},
$$

$\left\{v^{\epsilon}\right\}$ can be decomposed into a weakly convergent part and a strongly convergent part, and similarly for $w^{\epsilon}$ (also see the exposition in Evans [23]). For 
this, the advantage can be taken of the first-order differential constraints, the commutativity of the Green operator $\Delta^{-1}$ on $\mathbb{R}^{3}$ with divergence, gradient, and curl, and most crucially, the ellipticity of $\Delta$, so that, for $\left\{v^{\epsilon} \cdot w^{\epsilon}\right\}$, the pairing of the weakly convergent terms pass to the limits via integration by parts, and the pairings of other terms can be dealt with directly. Observe that the Laplace-Beltrami operator $\Delta$ defined for differential forms on any oriented closed Riemannian manifold $(M, g)$ is always elliptic, and it has a decomposition similar to (2.1):

$$
\Delta=d \circ \delta+\delta \circ d
$$

where $d$ is the exterior differential and $\delta$ is its $L^{2}$-adjoint ( $c f . \S 6$ in [53] for the details), so that the div-curl lemma is ready to be generalized to Riemannian manifolds.

The third approach, which is the main content of this section, is functionalanalytic. As aforementioned, the existing div-curl lemmas are formulated in terms of vector fields or local differential forms on Euclidean spaces ( $c f$. [16, 23. [45, 39, 50]), and some generalizations to Riemannian manifolds are available (cf. [11, 31, 33). For example, Kozono-Yanagisawa 33 obtained a div-curl lemma using functional-analytic results on $L^{2}\left(\mathbb{R}^{n}\right)$, as well as a geometric version, with emphasis on the weak convergence of vector fields up to the boundary of the domain or compact Riemannian manifold, which requires the divergence and curl of the vector fields to be bounded in $L^{2}$. One of our key observations is that, for the "usual" div-curl lemmas - with the exception of certain end-point cases, e.g., Theorem 2.3. the specific geometry of Euclidean spaces or manifolds plays no essential role. Based on this observation, we have formulated and established a general compensated compactness theorem through bounded linear operators on Banach spaces in [11]. Roughly speaking, it may be stated as follows: If two bounded linear operators $S$ and $T$ between Banach spaces satisfy two conditions: One is algebraic ( $S$ and $T$ are orthogonal to each other), and the other is analytic ( $S \oplus T$ determines nearly everything), then a result in the spirit of Lemma 1.1 holds, with div and curl replaced by $S$ and $T$, respectively.

We now discuss the functional-analytic compensated compactness theorem in Banach spaces, as well as its geometric implications. For some background on functional analysis, we refer to [25].

Let us first explain some notations: In the sequel, $\mathcal{H}$ is a Hilbert space over the field $\mathbb{K}=\mathbb{R}$ or $\mathbb{C}$ so that $\mathcal{H}=\mathcal{H}^{*}$, and $Y, Z$ are two Banach spaces over $\mathbb{K}$. We use $\mathcal{H}^{*}$ and $Y^{*}, Z^{*}, \ldots$, to denote the dual Hilbert and Banach spaces, respectively. In what follows, we consider the bounded linear operators:

$$
S: \mathcal{H} \rightarrow Y, \quad T: \mathcal{H} \rightarrow Z .
$$

For their adjoint operators, we write

$$
S^{\dagger}: Y^{*} \rightarrow \mathcal{H} \quad T^{\dagger}: Z^{*} \rightarrow \mathcal{H} .
$$


By $\langle\cdot, \cdot\rangle_{Y},\langle\cdot, \cdot\rangle_{Z}, \ldots$, we mean the duality pairings on suitable Banach spaces, and notation $\langle\cdot, \cdot\rangle$ without subscripts is reserved for the inner product on $\mathcal{H}$. Furthermore, for any normed vector spaces $X, X_{1}$, and $X_{2}$, we write $\left\{s^{\epsilon}\right\} \subset X$ for a sequence $\left\{s^{\epsilon}\right\}$ in $X$ as a subset, and $X_{1} \Subset X_{2}$ for a compact embedding between the normed vector spaces. We use $\|\cdot\|_{X}$ to denote the norm in $X$, write $\rightarrow$ for the strong convergence of sequences under the norm, and write $\rightarrow$ for the weak convergence. $\bar{B}_{X}:=\{x \in X:\|x\| \leq 1\}$ is the closed unit ball in $X$, and $B_{X}:=\{x \in X:\|x\|<1\}$ is the open unit ball. Moreover, for a linear operator $L: X_{1} \mapsto X_{2}$, its kernel is written as $\operatorname{ker}(L) \subset X_{1}$, and its range is $\operatorname{ran}(L) \subset X_{2}$. Finally, for $X_{1} \subset X$ as a vector subspace, its annihilator is defined as $X_{1}^{\perp}:=\left\{f \in X^{*}: f(x)=0\right.$ for all $\left.x \in X_{1}\right\}$.

To proceed, we define the following linear operators:

$$
\begin{cases}S \oplus T: \mathcal{H} \mapsto Y \oplus Z, & (S \oplus T) h:=(S h, T h) ; \\ S^{\dagger} \vee T^{\dagger}:(Y \oplus Z)^{*} \cong Y^{*} \oplus Z^{*} \mapsto \mathcal{H}, & \left(S^{\dagger} \vee T^{\dagger}\right)(a, b):=S^{\dagger} a+T^{\dagger} b\end{cases}
$$

for $h \in \mathcal{H}, a \in Y^{*}$, and $b \in Z^{*}$. The direct sum $Y \oplus Z$ is always endowed with the norm: $\|(y, z)\|_{Y} \oplus Z:=\|y\|_{Y}+\|z\|_{Z}$. Also, it is direct to see that $(S \oplus T)^{\dagger}=S^{\dagger} \vee T^{\dagger}$.

Our compensated compactness theorem is formulated in the following:

Theorem 2.1 (Theorem 2.1] in [11]). Let $\mathcal{H}=\mathcal{H}^{*}$ be a Hilbert space over $\mathbb{K}$, $Y$ and $Z$ be reflexive Banach spaces over $\mathbb{K}$, and $S: \mathcal{H} \mapsto Y$ and $T: \mathcal{H} \mapsto Z$ be bounded linear operators satisfying

(Op 1) Orthogonality:

$$
S \circ T^{\dagger}=0, \quad T \circ S^{\dagger}=0 ;
$$

(Op 2) For some Hilbert space $\left(\mathcal{H} ;\|\cdot\|_{\mathcal{H}}\right)$ such that $\mathcal{H}$ embeds compactly into $\mathcal{H}$, there exists a constant $C>0$ so that, for all $h \in \mathcal{H}$,

$$
\|h\|_{\mathcal{H}} \leq C\left(\|(S h, T h)\|_{Y} \bigoplus^{Z}+\|h\|_{\mathcal{H}}\right)=C\left(\|S h\|_{Y}+\|T h\|_{Z}+\|h\|_{\mathcal{H}}\right) .
$$

Assume that two sequences $\left\{u^{\epsilon}\right\},\left\{v^{\epsilon}\right\} \subset \mathcal{H}$ satisfy the following conditions:

(Seq 1) $u^{\epsilon} \rightarrow \bar{u}$ and $v^{\epsilon} \rightarrow \bar{v}$ in $\mathcal{H}$ as $\epsilon \rightarrow 0$;

(Seq 2) $\left\{S u^{\epsilon}\right\}$ is pre-compact in $Y$, and $\left\{T v^{\epsilon}\right\}$ is pre-compact in $Z$.

Then

$$
\left\langle u^{\epsilon}, v^{\epsilon}\right\rangle \rightarrow\langle\bar{u}, \bar{v}\rangle \quad \text { as } \epsilon \rightarrow 0 .
$$

Outline of Proof. We now sketch the main steps of the proof here. The interested readers are referred to [11] for the details.

Step 1. Claim: $S \oplus T: \mathcal{H} \mapsto Y \oplus Z$ has finite-dimensional kernel and closed range. As $Y$ and $Z$ are reflexive, $\operatorname{ran}(S \oplus T)$ is also a reflexive Banach 
space. This observation guarantees that all the assumptions (Op 1)-(Op 2) and (Seq 1)-(Seq 2) remain valid, provided that $Y \oplus Z$ is replaced by $\operatorname{ran}(S \oplus T)$, i.e., $S$ and $T$ are surjective. Thus, once Step 1 has been established, we can assume that $S \oplus T$ is Fredholm in the subsequent arguments.

Indeed, to show $\operatorname{dim}_{\mathbb{K}} \operatorname{ker}(S \oplus T)<\infty$, by the classical Riesz lemma, it suffices to check that the closed unit ball of $\operatorname{ker}(S \oplus T)$ is compact in the norm topology of $Y \oplus Z$. To this end, let $j: \mathcal{H} \hookrightarrow \mathcal{H}$ be the compact embedding in (Op 2). Then, for any $h \in \mathcal{H}$ such that $j(h) \in j[\operatorname{ker}(S \oplus T)] \cap \bar{B}_{\mathcal{H}}$, the same condition yields

$$
\|h\|_{\mathcal{H}} \leq C\left(\|S h\|_{Y}+\|T h\|_{Z}+\|j(h)\|_{\mathcal{H}}\right) \leq C .
$$

Therefore, the unit ball of $j[\operatorname{ker}(S \oplus T)]$ in $\mathcal{H}$ is finite-dimensional, and the same conclusion holds for $\operatorname{ker}(S \oplus T)$ as $j$ is an embedding.

To show $\operatorname{ran}(S \oplus T) \subset Y \oplus Z$ as a closed subspace, we take any sequence $\left\{h^{\mu}\right\} \subset \mathcal{H}$ such that $(S \oplus T) h^{\mu} \rightarrow w$ in the norm topology of $Y \oplus Z$ and argue that $w \in \operatorname{ran}(S \oplus T)$. This follows from the following coercivity estimate: There exists a universal constant $\epsilon_{0}>0$ such that

$$
\|(S \oplus T) h\|_{Y} \oplus Z Z \epsilon_{0}\|j(h)\|_{\mathcal{H}} \quad \text { for all } h \in \mathcal{H} .
$$

The estimate in (2.6) is obtained via a contradiction argument, by taking into account of the finite-dimensionality of $\operatorname{ker}(S \oplus T)$ and the 1-homogeneity of (2.6). Then we decompose $h^{\mu}=k^{\mu}+r^{\mu}$ for $k^{\mu} \in \operatorname{ker}(S \oplus T)$ and $r^{\mu} \in$ $[\operatorname{ker}(S \oplus T)]^{\perp}$. In view of the inequality:

$$
\left\|(S \oplus T)\left(h^{\mu_{1}}-h^{\mu_{2}}\right)\right\|_{Y \oplus Z} \geq \epsilon_{0}\left\|j\left(r^{\mu_{1}}-r^{\mu_{2}}\right)\right\|_{\mathcal{H}},
$$

we find that $\left\{j\left(r^{\mu}\right)\right\}$ is a Cauchy sequence in $\mathcal{H}$, which converges to some $j(r)$. Then it is direct to check that $(S \oplus T) r=w$, which leads to the claim in Step 1.

Notice in passing that we have obtained the following decomposition of $\mathcal{H}$ along $S \oplus T$ :

$$
\mathcal{H}=\operatorname{ker}(S \oplus T) \bigoplus \operatorname{ran}\left(S^{\dagger} \vee T^{\dagger}\right),
$$

where $\bigoplus$ is the topological direct sum of the Banach spaces, with the summands being orthogonal with respect to the inner product on $\mathcal{H}$. Moreover, note that only the analytic assumption (Op 2) on $S$ and $T$ has been used in Step 1.

Step 2. From now on, $S \oplus T$ is assumed to be surjective and with finitedimensional kernel. In this step, we decompose each of the two sequences $\left\{v^{\epsilon}\right\}$ and $\left\{w^{\epsilon}\right\}$ into three parts: an $S$-free part, a $T$-free part, and a remainder in the finite-dimensional space $\operatorname{ker}(S \oplus T)$. This is done via the generalized Laplacian. 
Indeed, we define operator $\phi: Y^{*} \oplus Z^{*} \mapsto Y \oplus Z$ as follows:

$$
\Delta:=(S \oplus T) \circ\left(S^{\dagger} \vee T^{\dagger}\right)=S S^{\dagger} \oplus T T^{\dagger} .
$$

Then, thanks to Eq. (2.7) and $\operatorname{ker}\left(S^{\dagger} \vee T^{\dagger}\right)=[\operatorname{ran}(S \oplus T)]^{\perp}$, we find that $\Delta$ also has finite-dimensional kernel and closed range and, as in Step 1, $\Delta$ can be assumed to be surjective. Denote by $\pi_{1}: \mathcal{H}=\operatorname{ker}(S \oplus T) \oplus \operatorname{ran}\left(S^{\dagger} \vee T^{\dagger}\right) \mapsto$ $\operatorname{ker}(S \oplus T)$ the canonical projection onto the first coordinate, which is a finiterank (hence compact) operator. Then our decomposition of $\left\{v^{\epsilon}\right\}$ and $\left\{w^{\epsilon}\right\}$ are given as follows:

$$
\begin{cases}u^{\epsilon}=\pi_{1}\left(u^{\epsilon}\right)+S^{\dagger} a^{\epsilon}+T^{\dagger} b^{\epsilon}, & v^{\epsilon}=\pi_{1}\left(v^{\epsilon}\right)+S^{\dagger} \tilde{a}^{\epsilon}+T^{\dagger} \tilde{b}^{\epsilon}, \\ \bar{u}=\pi_{1}(\bar{u})+S^{\dagger} a+T^{\dagger} b, & \bar{v}=\pi_{1}(\bar{v})+S^{\dagger} \tilde{a}+T^{\dagger} \tilde{b},\end{cases}
$$

for some $a, \tilde{a}, a^{\epsilon}, \tilde{a}^{\epsilon} \in Y^{*}$ and $b, \tilde{b}, b^{\epsilon}, \tilde{b}^{\epsilon} \in Z^{*}$. Applying the algebraic condition (Op 1) of operators $S$ and $T$, the inner products become:

$$
\left\{\begin{array}{l}
\left\langle u^{\epsilon}, v^{\epsilon}\right\rangle=\left\langle\pi_{1}\left(u^{\epsilon}\right), \pi_{1}\left(v^{\epsilon}\right)\right\rangle+\left\langle S^{\dagger} a^{\epsilon}, S^{\dagger} \tilde{a}^{\epsilon}\right\rangle+\left\langle T^{\dagger} b^{\epsilon}, T^{\dagger} \tilde{b}^{\epsilon}\right\rangle, \\
\langle\bar{u}, \bar{v}\rangle=\left\langle\pi_{1}(\bar{u}), \pi_{1}(\bar{v})\right\rangle+\left\langle S^{\dagger} a, S^{\dagger} \tilde{a}\right\rangle+\left\langle T^{\dagger} b, T^{\dagger} \tilde{b}\right\rangle .
\end{array}\right.
$$

Owing to the compactness of $\pi_{1},\left\langle\pi_{1}\left(u^{\epsilon}\right), \pi_{1}\left(v^{\epsilon}\right)\right\rangle \rightarrow\left\langle\pi_{1}(\bar{u}), \pi_{1}(\bar{v})\right\rangle$ as $\epsilon \rightarrow 0$. Therefore, to conclude the theorem, it remains to establish

$$
\left\langle S^{\dagger} a^{\epsilon}, S^{\dagger} \tilde{a}^{\epsilon}\right\rangle+\left\langle T^{\dagger} b^{\epsilon}, T^{\dagger} \tilde{b}^{\epsilon}\right\rangle \rightarrow\left\langle S^{\dagger} a, S^{\dagger} \tilde{a}\right\rangle+\left\langle T^{\dagger} b, T^{\dagger} \tilde{b}\right\rangle \quad \text { as } \epsilon \rightarrow 0,
$$

which is the content of the next step.

Step 3. To prove the convergence in (2.11), we start with the following two observations:

(i) The left-hand side of (2.11) can be expressed in terms of the generalized Laplacian:

$$
\begin{aligned}
& \left\langle S^{\dagger} a^{\epsilon}, S^{\dagger} \tilde{a}^{\epsilon}\right\rangle+\left\langle T^{\dagger} b^{\epsilon}, T^{\dagger} \tilde{b}^{\epsilon}\right\rangle \\
& =\left\langle S S^{\dagger} a^{\epsilon}, \tilde{a}^{\epsilon}\right\rangle_{Y}+\left\langle b^{\epsilon}, T T^{\dagger} \tilde{b}^{\epsilon}\right\rangle_{Z}=\left\langle\not \Delta\left(a^{\epsilon}, \tilde{b}^{\epsilon}\right),\left(\tilde{a}^{\epsilon}, b^{\epsilon}\right)\right\rangle_{Y} \bigoplus Z
\end{aligned}
$$

(ii) Multiplying $S$ to $u^{\epsilon}$ and $T$ to $v^{\epsilon}$ in (2.9) and invoking (Op 1), we have

$$
S u^{\epsilon}=S S^{\dagger} a^{\epsilon}, \quad T v^{\epsilon}=T T^{\dagger} \tilde{b}^{\epsilon},
$$

so that

$$
\Delta\left(a^{\epsilon}, \tilde{b}^{\epsilon}\right)=\left(S u^{\epsilon}, T v^{\epsilon}\right) .
$$

Now, as $\left\{S u^{\epsilon}\right\} \subset Y$ and $\left\{T v^{\epsilon}\right\} \subset Z$ are pre-compact by assumption (Seq 2 ), it suffices to show the boundedness of $\left\{\left(\tilde{a}^{\epsilon}, b^{\epsilon}\right)\right\}$ in the norm topology of $Y^{*} \oplus Z^{*}$ to reach the conclusion. Furthermore, in view of the specific form of 
the expression involved in (2.11), it is enough to exhibit one particular representative $\left(\tilde{a}^{\epsilon}, b^{\epsilon}\right)$ in the co-set $\boldsymbol{\Delta}^{-1}\left\{\left(S v^{\epsilon}, T u^{\epsilon}\right)\right\}$ such that $\left\|\left(\tilde{a}^{\epsilon}, b^{\epsilon}\right)\right\|_{Y^{*}} \bigoplus Z^{*} \leq$ $C$, where $C>0$ is independent of $\epsilon$. As $\left\{\left(S v^{\epsilon}, T u^{\epsilon}\right)\right\}$ is uniformly bounded in the norm topology of $Y \oplus Z$, owing to the weak convergence of $\left\{v^{\epsilon}\right\}$ and $\left\{w^{\epsilon}\right\}$ assumed in (Seq 1), the desired result follows from a standard result in functional analysis, which is Claim in the proof of Theorem 3.1 in [11]. This completes the proof.

With the benefit of hindsight, let us now explain the motivation for Theorem 2.1 and its relations with the earlier versions of the div-curl lemmas. Consider a 3-dimensional oriented closed manifold $M$ (differentiable, or of weaker Sobolev regularity, not necessarily Riemannian). We denote by $\Omega^{q}(M)$ the space of differential $q$-forms on $M$, by $*: \Omega^{q}(M) \mapsto \Omega^{\operatorname{dim}(M)-q}(M)$ the Hodge-star, by $d: \Omega^{q}(M) \mapsto \Omega^{q+1}(M)$ the exterior differential, and by $\sharp$ the tonic operator, i.e., the canonical isomorphism between the co-tangent bundle $T^{*} M$ and the tangent bundle $T M$ by raising indices in the coefficients. It is well-known that div, grad, and curl can be defined intrinsically via the commutative diagram:

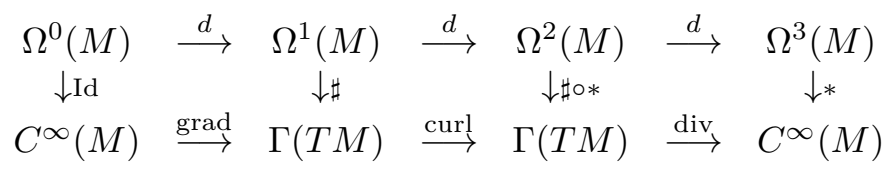

In particular, the Riemannian metric on $M$ plays no role at all. The "orthogonality" of div and curl in the sense of (Op 1) in Theorem 2.1, which follows from the cohomological chain condition $d \circ d=0, \delta \circ \delta=0$, is a purely algebraic relation. Therefore, it is not surprising that a compensated compactness theorem with greater generality and abstractness is available.

Moreover, the Hodge decomposition approach to the div-curl lemma initiated by Robbin-Rogers-Temple in [45] makes use of the Laplacian $\Delta$ on flat $\mathbb{R}^{3}$. If we take $S=\operatorname{div}, T=\operatorname{curl}, \mathcal{H}=L^{2}\left(\mathbb{R}^{3} ; \mathbb{R}^{3}\right), Y=H^{-1}\left(\mathbb{R}^{3} ; \mathbb{R}\right)$, and $\underline{\mathcal{H}}=Z=H^{-1}\left(\mathbb{R}^{3} ; \mathbb{R}^{3}\right)$ with suitable localizations if necessary, the classical div-curl lemma (Lemma 1.1) is immediately recovered. Our generalized Laplacian $\Delta$ extends the flat Laplacian and, more generally, the Laplace-Beltrami operator $\Delta$ on manifolds, in view of Eqs. (2.1) - (2.2). The Fredholmness of $\Delta$ follows from the Hodge decomposition theorem, cf. $\S 6$ in [53].

Before our subsequent development, we remark that the assumption of the reflexivity of $Y$ and $Z$ is crucial, since several counterexamples have been constructed for non-reflexive $Y$ and $Z$ (see [18] and Remark 3.2 in [11]).

Now we discuss a geometric consequence of Theorem 2.1. Using the expression for $\Delta=\Delta$ on Riemannian manifolds in terms of $S=d$ and $T=\delta$ as in Eq. (2.2), we have 
Theorem 2.2 (Geometrically intrinsic div-curl lemma A, Theorem 3.3 in [11). Let $(M, g)$ be an $n$-dimensional Riemannian manifold. Let $\left\{\omega^{\epsilon}\right\},\left\{\tau^{\epsilon}\right\} \subset$ $L_{\text {loc }}^{2}\left(M ; \wedge^{q} T^{*} M\right)$ be two families of differential $q$-forms such that

(i) $\omega^{\epsilon} \rightarrow \bar{\omega}$ and $\tau^{\epsilon} \rightarrow \bar{\tau}$ weakly in $L_{\mathrm{loc}}^{2}\left(M ; \bigwedge^{q} T^{*} M\right)$;

(ii) There are compact subsets of the corresponding Sobolev spaces, $K_{d}$ and $K_{\delta}$, such that

$$
\left\{\begin{array}{l}
\left\{d \omega^{\epsilon}\right\} \subset K_{d} \Subset H_{\mathrm{loc}}^{-1}\left(M ; \bigwedge^{q+1} T^{*} M\right), \\
\left\{\delta \tau^{\epsilon}\right\} \subset K_{\delta} \Subset H_{\mathrm{loc}}^{-1}\left(M ; \bigwedge^{q-1} T^{*} M\right) .
\end{array}\right.
$$

Then $\left\langle\omega^{\epsilon}, \tau^{\epsilon}\right\rangle$ converges to $\langle\bar{\omega}, \bar{\tau}\rangle$ in $\mathcal{D}^{\prime}(M)$, that is,

$$
\int_{M}\left\langle\omega^{\epsilon}, \tau^{\epsilon}\right\rangle \psi \mathrm{d} V_{g} \longrightarrow \int_{M}\langle\bar{\omega}, \bar{\tau}\rangle \psi \mathrm{d} V_{g} \quad \text { for any } \psi \in C_{c}^{\infty}(M) .
$$

Since the conclusion for the weak continuity in Theorem 2.2 is in the distributional sense, we may assume $M$ to be oriented and closed without loss of generality in the proof. Here and in the sequel, $W^{k, p}\left(M ; \wedge^{q} T^{*} M\right)$ denotes the Sobolev space of differential $q$-forms with $W^{k, p}$-regularity. Then $\Delta$ : $\Omega^{q}(M) \mapsto \Omega^{q}(M)$, as well as $\Delta: W^{k, p}\left(M ; \bigwedge^{q} T^{*} M\right) \mapsto W^{k-2, p}\left(M ; \bigwedge^{q} T^{*} M\right)$, for $0 \leq q \leq n=\operatorname{dim}(M)$, is elliptic, which is crucial for the verification of condition (Op 2) in Theorem 2.1. As is well-known, the analogous operator on semi-Riemannian manifolds is not elliptic in general, so that Theorem 2.2 may not be extended directly to the semi-Riemannian settings.

Next, we state an endpoint case of the above theorem, for which the firstorder differential constraints are prescribed in non-reflexive Banach spaces $W^{-1,1}$, in contrast to condition (Seq 2) in Theorem 2.1. The underlying argument for the proof essentially follows from that in Conti-Dolzmann-Müller ([18]), which employs a Lipschitz truncation argument and the pre-compactness theorems for $L^{1}$ (e.g., Chacon's biting lemma, the Dunford-Pettis theorem, etc.) to reduce to the reflexive case.

Theorem 2.3 (Geometrically intrinsic div-curl lemma B, Theorem 6.1 in [11]). Let $(M, g)$ be an $n$-dimensional manifold. Let $\left\{\omega^{\epsilon}\right\} \subset L_{\mathrm{loc}}^{2}\left(M ; \bigwedge^{q} T^{*} M\right)$ and $\left\{\tau^{\epsilon}\right\} \subset L_{\mathrm{loc}}^{2}\left(M ; \wedge^{q} T^{*} M\right)$ be two families of differential $q$-forms. Suppose that

(i) $\omega^{\epsilon} \rightarrow \bar{\omega}$ and $\tau^{\epsilon} \rightarrow \bar{\tau}$ weakly in $L_{\mathrm{loc}}^{2}\left(M ; \bigwedge^{q} T^{*} M\right)$ as $\epsilon \rightarrow 0$;

(ii) There are compact subsets of the corresponding Sobolev spaces, $K_{d}$ and $K_{\delta}$, such that

$$
\left\{\begin{array}{l}
\left\{d \omega^{\epsilon}\right\} \subset K_{d} \Subset W_{\mathrm{loc}}^{-1,1}\left(M ; \bigwedge^{q+1} T^{*} M\right), \\
\left\{\delta \tau^{\epsilon}\right\} \subset K_{\delta} \Subset W_{\mathrm{loc}}^{-1,1}\left(M ; \bigwedge^{q-1} T^{*} M\right) ;
\end{array}\right.
$$


(iii) $\left\{\left\langle\omega^{\epsilon}, \tau^{\epsilon}\right\rangle\right\}$ is equi-integrable.

Then $\left\langle\omega^{\epsilon}, \tau^{\epsilon}\right\rangle$ converges to $\langle\bar{\omega}, \bar{\tau}\rangle$ in $\mathcal{D}^{\prime}(M)$, that is,

$$
\int_{M}\left\langle\omega^{\epsilon}, \tau^{\epsilon}\right\rangle \psi \mathrm{d} V_{g} \longrightarrow \int_{M}\langle\bar{\omega}, \bar{\tau}\rangle \psi \mathrm{d} V_{g} \quad \text { for any } \psi \in C_{c}^{\infty}(M) .
$$

To conclude this section, we remark that the preceding intrinsic div-curl lemmas (Theorems 2.2 2.3) can be extended to the case of general Hölder exponents, namely that $\left\{\omega^{\epsilon}\right\} \subset L_{\mathrm{loc}}^{r}\left(M ; \bigwedge^{q} T^{*} M\right)$ and $\left\{\tau^{\epsilon}\right\} \subset L_{\mathrm{loc}}^{s}\left(M ; \bigwedge^{q} T^{*} M\right)$ with $\frac{1}{r}+\frac{1}{s}=1$. Since $L^{r}$ is not a Hilbert space unless $r=2$, such generalizations cannot be directly deduced from Theorem 2.1. Nevertheless, they follow from similar arguments, with slight modifications in light of Eq. (2.2). We refer to Theorems 3.7, Theorem 6.2, and Appendix in [11] for the details; also see $\S 5$ in 33 .

\section{Isometric Immersions of Riemannian Manifolds and the Gauss-Codazzi-Ricci (GCR) Equations}

In this section, we briefly discuss the geometric preliminaries for the isometric immersions of Riemannian manifolds. We restrain ourselves to the constructions directly related to our subsequent development. We refer to the classical texts [19, 22, 46] for more detailed treatments on differential geometry, to Han-Hong [30], as well as the classical papers by Nash [42, 43], for isometric immersions.

From now on, let $M$ be an $n$-dimensional Riemannian manifold, and let $g$ be a Riemannian metric on $M$. Motivated by the applications in nonlinear elasticity ( $c f . \quad 2,13,15,36$ ), we consider the metrics of weaker regularity: $g \in W_{\text {loc }}^{1, p}$ A $W_{\text {loc }}^{2, p} \operatorname{map} f:(M, g) \mapsto\left(\mathbb{R}^{n+k}, g_{0}\right)$ for the Euclidean metric $g_{0}$ is an immersion if the differential $d f_{P}$ is injective for each $P \in M$, and it is an embedding if $f$ itself is also injective. Moreover, $f$ is isometric if

$$
d f \otimes d f=g,
$$

that is, for any $P \in M$ and vector fields $X, Y$ on $M$,

$$
d f_{P}(X) \cdot d f_{P}(Y)=g_{P}(X, Y)
$$

where $g_{P}$ denotes the metric evaluated at $P$, and $\cdot$ is the Euclidean dot product on $\mathbb{R}^{n+k}$. Notice that Eq. (3.2) makes sense in the distributional sense when $p^{*}=\frac{n p}{n-p} \geq 2$, and that $g$ has a continuous representative when $p>n$, in view of the Sobolev embeddings $W^{2, p}\left(\mathbb{R}^{n}\right) \hookrightarrow W^{1, q}\left(\mathbb{R}^{n}\right)$ for $1 \leq q \leq p^{*}$ and $W^{1, p}\left(\mathbb{R}^{n}\right) \hookrightarrow C^{0}\left(\mathbb{R}^{n}\right)$ for $p>n$.

In differential geometry, the description of an isometric immersion is equivalent to the determination of how $\mathbb{R}^{n+k}$ - viewed as its own tangent spaces - 
can be split into the immersion-independent and immersion-dependent geometry of $M$. More precisely, for each point $P \in M$, we have the vector space direct sum

$$
\mathbb{R}^{n+k}=T_{P} M \bigoplus T_{P} M^{\perp},
$$

where $T_{P} M$ is the tangent space of $M$ at $P$, and $T_{P} M^{\perp}$ is its complement in $\mathbb{R}^{n+k}$, interpreted as the normal space.

To study the isometric immersions, two approaches have been employed from the PDE point of view. One is to deal directly with Eq. (3.2), which is a first-order, nonlinear, generally under-determined PDE; the other is to derive a PDE system by taking two more derivatives and solves for the compatibility conditions. The former approach has been employed by Nash [42, 43] to establish the existence of $C^{1}$ and $C^{k}$ isometric embeddings for large enough codimensions; also see Günther [29] for a simplification. For the latter approach, the compatibility conditions read schematically as follows:

$$
\begin{aligned}
0 & =\text { Curvature of } \mathbb{R}^{n+k} \\
& \Longleftrightarrow\left\{\begin{array}{l}
\text { Curvature in (tangential, tangential) direction }=0, \\
\text { Curvature in (tangential, normal) direction }=0, \\
\text { Curvature in (normal, normal) direction }=0 .
\end{array}\right.
\end{aligned}
$$

To continue, we use Latin letters $X, Y, Z, W, \ldots$ to denote the tangential vector fields in $\Gamma(T M)$, write Greek letters $\xi, \eta, \zeta, \ldots$ for the normal vector fields in $\Gamma\left(T M^{\perp}\right)$, and identify vector fields with first-order differential operators. Then $X Y$ and $X \xi$ are vector fields in $\Gamma(T M)$ and $\Gamma\left(T M^{\perp}\right)$, respectively. Let us consider the well-known geometric quantities:

- Immersion-independent quantities. Taking one derivative in $g$ leads to the Levi-Civita connection (or covariant derivative) $\nabla: \Gamma(T M) \times$ $\Gamma(T M) \mapsto \Gamma(T M)$, and taking one further derivative gives us the Riemann curvature tensor $R: \Gamma(T M) \times \Gamma(T M) \times \Gamma(T M) \times \Gamma(T M) \mapsto \mathbb{R}$.

- Immersion-dependent quantities. For given $\nabla, R$ as above, consider the isometric immersion $f:(M, g) \hookrightarrow\left(\mathbb{R}^{n+k}, g_{0}\right)$. We define the second fundamental form $B: \Gamma(T M) \times \Gamma(T M) \mapsto \Gamma\left(T M^{\perp}\right)$ by

$$
B(X, Y):=X Y-\nabla_{X} Y,
$$

and the normal connection $\nabla^{\perp}: \Gamma(T M) \times \Gamma\left(T M^{\perp}\right) \mapsto \Gamma\left(T M^{\perp}\right)$ by

$$
\nabla_{X}^{\perp} \xi:=\text { projection of } X \xi \text { (at each point) onto } T M^{\perp} \text {. }
$$

Then the right-hand sides of the schematic equations in ( $)$ can be expressed via the quantities $\left(g, \nabla, R, B, \nabla^{\perp}\right)$, resulting in the Gauss, Codazzi, and Ricci equations in (3.6), (3.7), and (3.8), respectively, below; see also Theorems $2.1-2.2$ in [11] and $\S 6$ in [19. 
Proposition 3.1. Suppose that $f \in W_{l o c}^{2, p}\left(M, g ; \mathbb{R}^{n+k}, g_{0}\right)$ for $p>n$ is an isometric immersion. Then the following compatibility conditions are satisfied in $\mathcal{D}^{\prime}$ :

$$
\begin{array}{rl}
\langle & B(X, W), B(Y, Z)\rangle-\langle B(Y, W), B(X, Z)\rangle=R(X, Y, Z, W) \\
X & B(Y, Z, \eta)-Y B(X, Z, \eta) \\
= & ([X, Y], Z, \eta)-B\left(X, \nabla_{Y} Z, \eta\right)-B\left(X, Z, \nabla_{Y}^{\perp} \eta\right) \\
& +B\left(Y, \nabla_{X} Z, \eta\right)+B\left(Y, Z, \nabla_{X}^{\perp} \eta\right) \\
X & \left\langle\nabla_{Y}^{\perp} \xi, \eta\right\rangle-Y\left\langle\nabla_{X}^{\perp} \xi, \eta\right\rangle \\
= & \left\langle\nabla_{[X, Y]}^{\perp} \xi, \eta\right\rangle-\left\langle\nabla_{X}^{\perp} \xi, \nabla_{Y}^{\perp} \eta\right\rangle+\left\langle\nabla_{Y}^{\perp} \xi, \nabla_{X}^{\perp} \eta\right\rangle \\
& +B\left(X \xi-\nabla_{X}^{\perp} \xi, Y, \eta\right)-B\left(X \eta-\nabla_{X}^{\perp} \eta, Y, \xi\right)
\end{array}
$$

where $X, Y, Z, W \in \Gamma(T M), \eta, \xi \in \Gamma\left(T M^{\perp}\right)$, and $[X, Y]=X Y-Y X$ is the Lie bracket. Here and in the sequel, we have used $\langle\cdot, \cdot\rangle$ to denote all the inner products induced by metrics, and $B(Y, Z, \eta):=\langle B(Y, Z), \eta\rangle$.

From the PDE perspectives, we view the immersion-dependent quantities $\left(B, \nabla^{\perp}\right)$ as to be solved, and the immersion-independent quantities $(g, \nabla, R)$ as being fixed. Indeed, in the isometric immersion problem, metric $g$ is prescribed, so are all the immersion-independent quantities; thus, the immersiondependent geometry determines the whole of the isometric immersion. Therefore, in the sequel, the GCR equations are always considered as a first-order nonlinear PDE system for $\left(B, \nabla^{\perp}\right)$.

Proposition 3.1 says that the GCR equations form a necessary condition for the existence of isometric immersions. The converse is known as the "realization problem" in elasticity: Given $\left(B, \nabla^{\perp}\right)$ satisfying the GCR equations, construct an isometric immersion (i.e., design an elastic body) whose immersiondependent geometry is prescribed by $\left(B, \nabla^{\perp}\right)$. This problem for both $C^{\infty}$ and $W_{\text {loc }}^{1, p}$ metrics has been answered in the affirmative, globally on simplyconnected manifolds; see Tenenblat [52] for the former, and Mardare [36, 37. and Szopos [49] for the latter. In [11, we adapt the geometric arguments in [52] to re-prove the realization theorem in $W_{\text {loc }}^{1, p}$ regularity, which simplifies the proofs in [36, 37, 49]. Moreover, this method sheds light on the weak rigidity problem of isometric immersions, which is the main content of $\S 4$.

Finally, we briefly sketch the main tool in [52] - the Cartan formalism - which serves as a bridge between the geometric problem of isometric immersions and the PDEs (GCR equations). In full generality, consider a vector bundle $E$ over $M$ of fibre $\mathbb{R}^{k}$, trivialized on a local chart $U \subset M$, i.e., $\left.E\right|_{U} \cong U \times \mathbb{R}^{k}$ as a diffeomorphism. Let $\left\{\partial_{i}\right\} \subset \Gamma(T U)$ be an orthonormal frame, and let $\left\{\omega^{i}\right\} \subset \Omega^{1}(U)$ be its dual (co-frame). Then we choose 
$\left\{\eta_{n+1}, \ldots, \eta_{n+k}\right\} \subset \Gamma(E)$ as an orthonormal basis for fibre $\mathbb{R}^{k}$, and set

$$
\begin{aligned}
& \omega_{j}^{i}\left(\partial_{k}\right):=\left\langle\nabla_{\partial_{k}} \partial_{j}, \partial_{i}\right\rangle, \\
& \omega_{\alpha}^{i}\left(\partial_{j}\right)=-\omega_{i}^{\alpha}\left(\partial_{j}\right):=\left\langle B\left(\partial_{i}, \partial_{j}\right), \eta_{\alpha}\right\rangle, \\
& \omega_{\beta}^{\alpha}\left(\partial_{j}\right):=\left\langle\nabla_{\partial_{j}}^{E} \eta_{\alpha}, \eta_{\beta}\right\rangle,
\end{aligned}
$$

where $\nabla^{E}$ is the bundle connection: $\nabla^{E}=\nabla^{\perp}$ for $E=T M^{\perp}=$ the normal bundle. All these constructions make sense in distributions for $g \in W_{\mathrm{loc}}^{1, p}$. Moreover, here and in the sequel, the following index convention is adopted:

$$
1 \leq i, j \leq n ; \quad 1 \leq a, b, c, d, e \leq n+k ; \quad n+1 \leq \alpha, \beta, \gamma \leq n+k .
$$

In this setting, the GCR equations on bundle $E$ are equivalent to the following two systems, known as the first and second structural equations of the Cartan formalism (cf. [52, 46, 47]):

$$
\begin{aligned}
& d \omega^{i}=\sum_{j} \omega^{j} \wedge \omega_{j}^{i}, \\
& d \omega_{b}^{a}=-\sum_{c} \omega_{b}^{c} \wedge \omega_{c}^{a}
\end{aligned}
$$

for each $i, a, b$. These equations can be represented compactly as first-order nonlinear Lie algebra-valued PDEs. Denoting by $\mathfrak{s o}(n+k)$ the Lie algebra of antisymmetric $(n+k) \times(n+k)$ matrices, we can write (3.12)-(3.13) as

$$
d w=w \wedge W, \quad d W+W \wedge W=0,
$$

where $W=\left\{w_{b}^{a}\right\} \in \Omega^{1}(U ; \mathfrak{s o}(n+k))$ which is known as the connection oneforms, $w=\left(\omega^{1}, \ldots, \omega^{n}, 0, \ldots, 0\right)^{\top} \in \Omega^{1}\left(U ; \mathbb{R}^{n+k}\right)$, and $\wedge$ operates by the wedge product on the factor of differential forms and matrix multiplication on the factor of the matrix Lie algebra, with respect to the factorization:

$$
\Omega^{1}(U ; \mathfrak{s o}(n+k)) \cong \Gamma\left(\bigwedge^{1} T^{*} U \bigotimes \mathfrak{s o}(n+k)\right) .
$$

In other words, the structural equations recast in (3.14) are also intrinsic, i.e., independent of the choice of local moving frames/coordinates $\left\{\partial_{i}\right\}$ and $\left\{\eta_{\alpha}\right\}$.

\section{Weak Rigidity of Isometric Immersions}

Finally, we discuss the weak rigidity of isometric immersions with weaker regularity in $W_{\text {loc }}^{2, p}$ as in the previous sections.

The rigidity problem of isometric immersions concerns the following: If $\left\{f^{\epsilon}\right\}$ is a sequence of isometric immersions of a manifold $M$ into $\left(\mathbb{R}^{n+k}, g_{0}\right)$, which 
converges to a map $f: M \mapsto \mathbb{R}^{n+k}$ in a certain topology, is $f$ still an isometric immersion? This problem has a history of celebrated results. Nash in [42] showed that the $C^{1}$ isometric immersions are not rigid. In particular, any $C^{\infty}$ short (i.e., distance-shrinking) immersion is $C^{0}$-close to an $C^{1}$ isometric immersion; see also [5] for a recent computer visualisation. In the same sense, Borisov in 4. proved that $C^{1, \alpha}$ isometric immersions are not rigid for $\alpha>$ 0 below a certain value, and this value has been improved in [17. On the other hand, the $C^{1, \alpha}$ isometric immersions for large enough $\alpha$ are classically known to be rigid; $c f$. [43] and the references therein. More recently, deep connections have been established between the transition phenomenon from the non-rigidity to rigidity of the $C^{1, \alpha}$ isometric immersions and Onsager's conjecture (concerning the dissipative weak solutions to the Euler equations in fluid dynamics). We refer the readers to [7] and the references cited therein for such developments.

Our focus is on the weak rigidity problem motivated by applications. In this case, for the sequence of isometric immersions $\left\{f^{\epsilon}\right\}$ that is weakly and locally convergent in $W^{2, p}$ for $p>n=\operatorname{dim}(M)$, we ask if the weak limit $\bar{f}$ is still a $W_{\text {loc }}^{2, p}$ isometric immersion. Indeed, we answer the question in the affirmative, thanks to the locally uniform $L^{p}$ bounds on the immersion-dependent geometry. This is in the spirit of the works by Langer [34] and the recent generalization by Breuning [3].

Our result can be formulated as follows:

Theorem 4.1 (Corollary 5.2 in [11]). Let $M$ be an n-dimensional simplyconnected Riemannian manifold with $W_{\mathrm{loc}}^{1, p}$ metric $g$ for $p>n$. Suppose that $\left\{f^{\epsilon}\right\}$ is a family of isometric immersions of $M$ into $\mathbb{R}^{n+k}$ with Euclidean metric, uniformly bounded in $W_{\text {loc }}^{2, p}\left(M ; \mathbb{R}^{n+k}\right)$, whose second fundamental forms and normal connections are $\left\{B^{\epsilon}\right\}$ and $\left\{\nabla^{\perp, \epsilon}\right\}$, respectively. Then, after passing to subsequences, $\left\{f^{\epsilon}\right\}$ converges to $\bar{f}$ weakly in $W_{\mathrm{loc}}^{2, p}$ which is still an isometric immersion $\bar{f}:(M, g) \rightarrow \mathbb{R}^{n+k}$. Moreover, the corresponding second fundamental form $\bar{B}$ is a weak limit of $\left\{B^{\epsilon}\right\}$, and the corresponding normal connection $\overline{\nabla^{\perp}}$ is a weak limit of $\left\{\nabla^{\perp, \epsilon}\right\}$, both taken in the weak topology in $L_{\mathrm{loc}}^{p}$.

Outline of Proof. We sketch the proof in three steps. For the details, we refer to $\S 5$ (Step 1), §4.2-§4.3 (Step 2), and §4.4 (Step 3) in [11].

Step 1. We show the equivalence between the existence of $W_{\text {loc }}^{2, p}$ isometric immersions and the existence of $W_{\text {loc }}^{1, p}$ solutions of the GCR equations in distributions (Proposition 3.1). Then the weak rigidity of isometric immersions is translated to the weak rigidity of the GCR equations.

Indeed, at the end of $\S 3$, it is remarked that the GCR equations are equivalent to the structural equations (3.14) of the Cartan formalism. Hence, by Proposition 3.1. Eq. (3.14) is a necessary condition for the existence of isometric immersions. Conversely, we follow the arguments in [52] to transform 
Eq. (3.14) into first-order nonlinear PDEs on Lie groups. More precisely, the isometric immersion $f$ satisfies the following equations (formulated as initial value problems) for $A \in W_{\text {loc }}^{1, p}(U ; O(n+k))$, where $O(n+k)$ is the group of $(n+k) \times(n+k)$ symmetric matrices:

$$
W=d A \cdot A^{\top}, \quad d f=w \cdot A .
$$

The above two equations are known as the Pfaff and the Poincaré systems. In the smooth case, they can be solved by the Frobenius theorem, by checking that the solution distribution is involutive. For the weak regularity case, we apply the theorems due to Mardare [36, 37] for the existence of solutions to Eq. (4.1). Then $d f \in W_{\mathrm{loc}}^{1, p} \hookrightarrow C_{\mathrm{loc}}^{0}$ for $n>p$, and it is non-degenerate and distance-preserving, thanks to the Poincaré system and the definition of $W$. This implies that $f$ is indeed an isometric immersion.

Step 2. The GCR equations in Proposition 3.1 are reformulated to manifest the div-curl structures, which admits the application of the intrinsic divcurl lemma, Theorem 2.2.

For this purpose, let us fix the tangential vector field $Z$ and normal vector fields $(\xi, \eta)$, and define the 2-tensor fields $V_{Z, \eta}^{(B)}, V_{\xi, \eta}^{\left(\nabla^{\perp}\right)}: \Gamma(T M) \times \Gamma(T M) \mapsto$ $\Gamma(T M)$ and 1-forms $\Omega_{Z, \eta}^{(B)}, \Omega_{\xi, \eta}^{\left(\nabla^{\perp}\right)}$ as follows:

$$
\begin{aligned}
& V_{Z, \eta}^{(B)}(X, Y):=B(X, Z, \eta) Y-B(Y, Z, \eta) X, \\
& V_{\xi, \eta}^{\left(\nabla^{\perp}\right)}(X, Y):=\left\langle\nabla_{Y}^{\perp} \xi, \eta\right\rangle X-\left\langle\nabla_{X}^{\perp} \xi, \eta\right\rangle Y, \\
& \Omega_{Z, \eta}^{(B)}:=-B(\bullet, Z, \eta), \\
& \Omega_{\xi, \eta}^{\left(\nabla^{\perp}\right)}:=\left\langle\nabla_{\bullet}^{\perp} \xi, \eta\right\rangle .
\end{aligned}
$$

For simplicity, we often drop the indices in both $\Omega$ and $V$ from now on.

To wit, these $\Omega$ 's are nothing but the contractions of $\left(B, \nabla^{\perp}\right)$, and the $V$ 's are obtained by applying $\Omega$ to the 2 -Grassmannian (i.e., the space of 2 planes) in $T M$ and polarized in the anti-symmetric fashion. Recall that the divergence can be defined intrinsically on manifolds by $\operatorname{div} X:=*\left(\mathcal{L}_{X} d V_{g}\right)$, where $\mathcal{L}$ denotes the Lie derivative, and the following well-known identities hold on manifolds:

$$
\left\{\begin{array}{l}
\mathcal{L}_{X}=d \circ \iota_{X}+\iota_{X} \circ d \quad \text { for } X \in \Gamma(T M), \\
d \alpha(X, Y)=X \alpha(Y)-Y \alpha(X)-\alpha([X, Y]) \quad \text { for } \alpha \in \Omega^{1}(M), X, Y \in \Gamma(T M) .
\end{array}\right.
$$

Thus, the divergence of $V$ 's and the generalized curl (i.e., $d)$ of $\Omega$ 's can be 
expressed as

$$
\begin{aligned}
\operatorname{div}\left(V_{Z, \eta}^{(B)}(X, Y)\right)= & Y B(X, Z, \eta)-X B(Y, Z, \eta) \\
& +B(X, Z, \eta) \operatorname{div} Y-B(Y, Z, \eta) \operatorname{div} X, \\
\operatorname{div}\left(V_{\xi, \eta}^{\left(\nabla^{\perp}\right)}(X, Y)\right)= & -Y\left\langle\nabla_{X}^{\perp} \xi, \eta\right\rangle+X\left\langle\nabla_{Y}^{\perp} \xi, \eta\right\rangle \\
& +\left\langle\nabla_{Y}^{\perp} \xi, \eta\right\rangle \operatorname{div} X-\left\langle\nabla_{X}^{\perp} \xi, \eta\right\rangle \operatorname{div} Y, \\
d\left(\Omega_{Z, \eta}^{(B)}\right)(X, Y)= & Y B(X, Z, \eta)-X B(Y, Z, \eta)+B([X, Y], Z, \eta), \\
d\left(\Omega_{\xi, \eta}^{\left(\nabla^{\perp}\right)}\right)(X, Y)= & -Y\left\langle\nabla_{X}^{\perp} \xi, \eta\right\rangle+X\left\langle\nabla_{Y}^{\perp} \xi, \eta\right\rangle-\left\langle\nabla_{[X, Y]}^{\perp} \xi, \eta\right\rangle,
\end{aligned}
$$

where the terms $B(X, Z, \eta) \operatorname{div} Y, B(Y, Z, \eta) \operatorname{div} X,\left\langle\nabla_{Y}^{\frac{1}{Y}} \xi, \eta\right\rangle \operatorname{div} X,\left\langle\nabla_{X}^{\frac{1}{X}} \xi, \eta\right\rangle \operatorname{div} Y$, $B([X, Y], Z, \eta)$, and $\left\langle\nabla_{[X, Y]}^{\perp} \xi, \eta\right\rangle$ are linear in $\left(B, \nabla^{\perp}\right)$, while the other terms on the right-hand sides of the above four equations involve first-order derivatives of $\left(B, \nabla^{\perp}\right)$. Moreover, for further development, it is crucial to observe that

$$
\left\{\begin{array}{l}
\operatorname{div}\left(V_{Z, \eta}^{(B)}(X, Y)\right)=d\left(\Omega_{Z, \eta}^{(B)}\right)(X, Y)+[\text { linear terms }], \\
\operatorname{div}\left(V_{\xi, \eta}^{\left(\nabla^{\perp}\right)}(X, Y)\right)=d\left(\Omega_{\xi, \eta}^{\left(\nabla^{\perp}\right)}\right)(X, Y)+[\text { linear terms }] .
\end{array}\right.
$$

Next, using the tensor fields $V$ and $\Omega$ introduced above, we can reformulate the GCR system as the following equations with emphasis on the pairings of $V$ 's and $\Omega$ 's:

$$
\begin{aligned}
& \sum_{\eta}\left\langle V_{Z, \eta}^{(B)}(X, Y), \Omega_{W, \eta}^{(B)}\right\rangle=R(X, Y, Z, W) \\
& d\left(\Omega_{Z, \eta}^{(B)}\right)(X, Y)+\sum_{\beta}\left\langle V_{\eta, \beta}^{\left(\nabla^{\perp}\right)}(X, Y), \Omega_{Z, \beta}^{(B)}\right\rangle \\
& \quad+B\left(Y, \nabla_{X} Z, \eta\right)-B\left(X, \nabla_{Y} Z, \eta\right)=0 \\
& \quad d\left(\Omega_{\xi, \eta}^{\left(\nabla^{\perp}\right)}\right)(X, Y)+\sum_{\beta}\left\langle V_{\eta, \beta}^{\left(\nabla^{\perp}\right)}(X, Y), \Omega_{\xi, \beta}^{\left(\nabla^{\perp}\right)}\right\rangle=\sum_{Z}\left\langle V_{Z, \xi}^{(B)}(X, Y), \Omega_{Z, \eta}^{(B)}\right\rangle,
\end{aligned}
$$

where all the summations are at most countable and locally finite.

Therefore, we have transformed the GCR equations in Proposition 3.1 into Eqs. (4.7)-(4.9), expressed in terms of the tensor fields $V$ and $\Omega$. Furthermore, the divergence of $V$ roughly equals to the generalized curl of the corresponding $\Omega$, which involves the derivatives of solutions $\left(B, \nabla^{\perp}\right)$ up to the first order.

Step 3. Now we are at the stage of applying the geometrically intrinsic div-curl lemma (Theorem 2.2) to conclude the weak rigidity of isometric immersions. Let $\left\{B^{\epsilon}, \nabla^{\perp, \epsilon}\right\}$ be the second fundamental forms and normal connections associated to the sequence of isometric immersions $\left\{f^{\epsilon}\right\}$. As $\left\{f^{\epsilon}\right\}$ is uniformly bounded in $W_{\text {loc }}^{2, p}$, the tensor fields $\left\{V^{\left(B^{\epsilon}\right)}, \Omega^{\left(B^{\epsilon}\right)}, V^{\left(\nabla^{\perp, \epsilon}\right)}, \Omega^{\left(\nabla^{\perp, \epsilon}\right)}\right\}$ 
are uniformly bounded in $L_{\mathrm{loc}}^{p}$, so that they are pre-compact in the weak topology. In view of Eqs. (4.8)-(4.9) and (4.6), the Cauchy-Schwarz inequality immediately yields that $\left\{\operatorname{div} V^{\left(B^{\epsilon}\right)}, d \Omega^{\left(B^{\epsilon}\right)}, \operatorname{div} V^{\left(\nabla^{\perp, \epsilon}\right)}, d \Omega^{\left(\nabla^{\perp, \epsilon}\right)}\right\}$ are uniformly bounded in $L_{\text {loc }}^{p / 2}$, which compactly embeds into $W_{\text {loc }}^{-1, p^{\prime}}$ for some $1<p^{\prime}<2$. On the other hand, they are uniformly bounded in $W_{\text {loc }}^{-1, p}$ for $p>n \geq 2$. Thus, by interpolation, we find that

$$
\left\{\operatorname{div} V^{\left(B^{\epsilon}\right)}, d \Omega^{\left(B^{\epsilon}\right)}, \operatorname{div} V^{\left(\nabla^{\perp, \epsilon}\right)}, d \Omega^{\left(\nabla^{\perp, \epsilon}\right)}\right\} \quad \text { are pre-compact in } H_{\text {loc }}^{-1},
$$

which is precisely the desired first-order differential constraints for the geometrically intrinsic div-curl lemma, Theorem 2.2 .

Therefore, applying Theorem 2.2, we obtain the following subsequential convergence results in $\mathcal{D}^{\prime}(M)$ :

$$
\begin{aligned}
& \left\langle V_{W, \eta}^{\left(B^{\epsilon}\right)}(X, Y), \Omega_{Z, \eta}^{\left(B^{\epsilon}\right)}\right\rangle \longrightarrow\left\langle V_{W, \eta}^{(B)}(X, Y), \Omega_{Z, \eta}^{(B)}\right\rangle, \\
& \left\langle V_{\eta, \beta}^{\left(\nabla^{\perp, \epsilon}\right)}(X, Y), \Omega_{\xi, \beta}^{\left(\nabla^{\perp, \epsilon}\right)}\right\rangle \longrightarrow\left\langle V_{\eta, \beta}^{\left(\nabla^{\perp}\right)}(X, Y), \Omega_{\xi, \beta}^{\left(\nabla^{\perp}\right)}\right\rangle, \\
& \left\langle V_{Z, \xi}^{\left(B^{\epsilon}\right)}(X, Y), \Omega_{Z, \eta}^{\left(B^{\epsilon}\right)}\right\rangle \longrightarrow\left\langle V_{Z, \xi}^{(B)}(X, Y), \Omega_{Z, \eta}^{(B)}\right\rangle, \\
& \left\langle V_{\eta, \beta}^{\left(\nabla^{\perp, \epsilon}\right)}(X, Y), \Omega_{Z, \beta}^{\left(B^{\epsilon}\right)}\right\rangle \longrightarrow\left\langle V_{\eta, \beta}^{\left(\nabla^{\perp}\right)}(X, Y), \Omega_{Z, \beta}^{(B)}\right\rangle,
\end{aligned}
$$

so that we can pass to the limits in Eqs. (4.7)-(4.9). As shown in Step 2 , these equations are equivalent to the GCR equations, which leads to the weak continuity of the GCR equations. Finally, by Step 1, we know that the existence of solutions of the GCR equations in $\mathcal{D}^{\prime}(M)$ are equivalent to the existence of isometric immersions in $W_{\mathrm{loc}}^{2, p}\left(M ; \mathbb{R}^{n+k}\right)$. Thus, the assertion is proved on the local trivialized chart $U \subset M$ as in Step 1. When $M$ is simplyconnected, we can pass from the local to global by a standard monodromy argument.

We now make three comments on our main theorem, Theorem 4.1 .

First of all, in Step 1, we have given a geometrically intrinsic proof of the realization theorem. This can be summarized as follows:

Corollary 4.2 (Theorem 5.2 in [11]). Let $(M, g)$ be an n-dimensional, simplyconnected Riemannian manifold with metric $g \in W_{\mathrm{loc}}^{1, p}$ for $p>n$, and let $\left(E, M, \mathbb{R}^{k}\right)$ be a vector bundle over $M$. Assume that $E$ has a $W_{\mathrm{loc}}^{1, p}$ metric $g^{E}$ and an $L_{\mathrm{loc}}^{p}$ connection $\nabla^{E}$ such that $\nabla^{E}$ is compatible with $g^{E}$. Moreover, suppose that there is an $L_{\mathrm{loc}}^{p}$ tensor field $S: \Gamma(E) \times \Gamma(T M) \rightarrow \Gamma(T M)$ satisfying

$$
\left\langle X, S_{\eta}(Y)\right\rangle-\left\langle S_{\eta}(X), Y\right\rangle=0
$$

and a corresponding $L_{\mathrm{loc}}^{p}$ tensor field $B: \Gamma(T M) \times \Gamma(T M) \rightarrow \Gamma(E)$ defined by

$$
\langle B(X, Y), \eta\rangle=-\left\langle S_{\eta}(X), Y\right\rangle .
$$

Then the following are equivalent: 
(i) The GCR equations as in Proposition 3.1 with $R^{\perp}$ replaced by $R^{E}$, the Riemann curvature operator on the bundle;

(ii) The Cartan formalism;

(iii) The existence of a global isometric immersion $f \in W_{\mathrm{loc}}^{2, p}\left(M ; \mathbb{R}^{n+k}\right)$ such that the induced normal bundle $T(f M)^{\perp}$, normal connection $\nabla^{\perp}$, and second fundamental form can be identified with $E, \nabla^{E}$, and $B$, respectively.

In (i)-(ii), the equalities are taken in the distributional sense and, in (iii), the isometric immersion $f \in W_{\mathrm{loc}}^{2, p}$ is unique a.e., modulo the Euclidean group of rigid motions $\mathbb{R}^{n+k} \rtimes O(n+k)$.

In view of the above corollary, for the purpose of weak rigidity, it is more natural to investigate the Cartan formalism. In particular, the GCR equations are recast into the compact identity $d W=W \wedge W$, which is the second structural equation (as in well-known in geometry, the first structural equation expresses the torsion-free property of the Levi-Civita connection). However, notice that the connection 1-form $W$ consists of only $\left(B, \nabla^{\perp}\right)$ so that, for the sequence of isometric immersions $\left\{f^{\epsilon}\right\}$ uniformly bounded in $W_{\text {loc }}^{2, p}\left(M ; \mathbb{R}^{n+k}\right)$, the corresponding $\left\{W^{\epsilon}\right\}$ is uniformly bounded in $L_{\mathrm{loc}}^{p}(M ; \mathfrak{s o}(n+k))$. Let $\bar{W}$ be a weak limit of $\left\{W^{\epsilon}\right\}$. Then, via similar arguments as in Steps 2-3 in the proof of Theorem 4.1, we can pass the limits in

$$
d W^{\epsilon}=W^{\epsilon} \wedge W^{\epsilon} \quad \Longrightarrow \quad d \bar{W}=\bar{W} \wedge \bar{W} .
$$

Therefore, applying Corollary 4.2, we obtain a simplified proof of Theorem 4.1

Second, for the most physically relevant case of the isometric immers$\mathrm{ing}$ /embedding of a 2-dimensional manifold (i.e., a surface) into $\mathbb{R}^{3}$, we can also establish the weak rigidity of the GCR equations in the critical case $p=n=2$ (where the Ricci equation is trivial). This is because, on the right-hand side of the Gauss equation (3.6), we have the Gauss curvature $R(X, Y, Z, W)$, which is a fixed $L^{1}$ function in the setting of isometric immersions, thanks to the Cauchy-Schwarz inequality. Therefore, it is equi-integrable. Then we can apply the critical case of the div-curl lemma (Theorem 2.3).

Corollary 4.3 (Theorem 6.3 in [11]). Let $M$ be a 2-dimensional, simplyconnected surface, and let $g$ be a metric in $H_{\mathrm{loc}}^{1}$. If $\left\{f^{\epsilon}\right\}$ is a family of $H_{\mathrm{loc}}^{2}$ isometric immersions of $M$ into $\mathbb{R}^{3}$ such that the corresponding second fundamental forms $\left\{B^{\epsilon}\right\}$ are uniformly $L^{2}$-bounded. Then, after passing to a subsequence, $\left\{f^{\epsilon}\right\}$ converges to $\bar{f}$ weakly in $H_{\mathrm{loc}}^{2}$ which is still an isometric immersion $\bar{f}:(M, g) \rightarrow \mathbb{R}^{3}$. Moreover, the corresponding second fundamental form $\bar{B}$ is a limit point of $\left\{B^{\epsilon}\right\}$ in the $L_{\text {loc }}^{2}$ topology.

Finally, it is easy to derive a slightly more general version of the weak rigidity theorem, Theorem 4.1, by allowing the metrics to be unfixed and 
strongly convergent in $W_{\text {loc }}^{1, p}$ for $p>n$. Such scenarios naturally arise in the regularization of a singular metric into smooth ones; cf. §7.2 in [11.

We remark in passing that the analogies of Theorem 4.1 and Corollaries 4.2 4.3 for isometric immersions into semi-Euclidean spaces of semi-Riemannian submanifolds (i.e., the metrics are non-degenerate, but may no longer be positive-definite; see O'Neill [44) are also valid. For the possibly degenerate hypersurfaces, using the machinery of rigging fields ( $c f$. 448, 35, 38), a counterpart of the Cartan formalism can be established, which leads to the weak rigidity, provided that the rigging fields are uniformly $L_{\mathrm{loc}}^{p}$ bounded. For a rigorous formulation and the proof of these results, see our forthcoming paper [12.

As discussed above, we have established the weak rigidity of isometric immersions (Theorem 4.1) in [11]. It would be interesting to explore its relation with the rigidity/non-rigidity results in stronger topologies (see the discussion at the beginning of $\S 4$ ), to extend it to the larger framework of the h-principle laid down by Gromov ([28]), and to examine what the possible implications are in fluid dynamics, in view of the connections between isometric immersions and Euler equations (see [1, 13] and [7]).

Acknowledgement. Gui-Qiang Chen's research was supported in part by the UK EPSRC Science and Innovation Award to the Oxford Centre for Nonlinear PDE (EP/E035027/1), the UK EPSRC Award to the EPSRC Centre for Doctoral Training in PDEs (EP/L015811/1), and the Royal Society-Wolfson Research Merit Award (UK). Siran Li's research was supported in part by the UK EPSRC Science and Innovation Award to the Oxford Centre for Nonlinear $\mathrm{PDE}(\mathrm{EP} / \mathrm{E} 035027 / 1)$.

\section{References}

[1] A. Acharya, G.-Q. Chen, S. Li, M. Slemrod, and D. Wang, Fluids, elasticity, geometry, and the existence of wrinkled solutions, Arch. Rational Mech. Anal. 226 (2017), 1009-1060.

[2] J. M. Ball, Convexity conditions and existence theorems in nonlinear elasticity, Arch. Rational Mech. Anal. 63 (1977), 337-403.

[3] P. Breuning, Immersions with bounded second fundamental form, J. Geom. Anal. 25 (2015), 1344-1386.

[4] Yu. F. Borisov, $C^{1, \alpha}$-isometric immersions of Riemannian spaces, Dokl. Akad. Nauk. SSSR, 163 (1965), 869-871.

[5] V. Borrelli, S. Jabrane, F. Lazarus, and B. Thibert, Flat tori in three-dimensional space and convex integration, Proc. Nat. Acad. Sci., 109 (2012), 7218-7223.

[6] R. L. Bryant, P. A. Griffiths, and D. Yang, Characteristics and existence of isometric embeddings, Duke Math. J. 50 (1983), 893-994. 
[7] T. Buckmaster, C. De Lellis, P. Isett, and L. Székelyhidi Jr., Anomalous dissipation for $\frac{1}{5}$-Hölder Euler flows, Ann. of Math. 182 (2015), 127-172.

[8] E. Cartan, Sur la possibilité de plonger un espace Riemannian donné dans un espace Euclidien, Ann. Soc. Pol. Math. 6 (1927), 1-7.

[9] G.-Q. Chen, J. Clelland, M. Slemrod, D. Wang, and D. Yang, Isometric embedding via strongly symmetric positive systems, Asian J. Math. (2018) (to appear). arXiv:1502.04356 (2015).

[10] G.-Q. Chen, H. Holden, and K. Karlsen, Hyperbolic Conservation Laws and Related Analysis with Applications, Springer-Verlag: Berlin-Heidelberg, 2014.

[11] G.-Q. Chen and S. Li, Global weak rigidity of the Gauss-Codazzi-Ricci equations and isometric immersions of Riemannian manifolds with lower regularity, J. Geom. Anal. (2018) (to appear). https://doi.org/10.1007/s12220-017-9893-1. ArXiv preprint: 1607.06862 (2016).

[12] G.-Q. Chen and S. Li, On the isometric immersions of semi-Riemannian submanifolds and general hypersurfaces: Lower regularity and weak rigidity, Preprint, October 2017.

[13] G.-Q. Chen, M. Slemrod, and D. Wang, Isometric immersions and compensated compactness, Commun. Math. Phys. 294 (2010), 411-437.

[14] G.-Q. Chen, M. Slemrod, and D. Wang, Weak continuity of the Gauss-CodazziRicci system for isometric embedding, Proc. Amer. Math. Soc. 138 (2010), 18431852.

[15] P. G. Ciarlet, L. Gratie, and C. Mardare, A new approach to the fundamental theorem of surface theory, Arch. Rational Mech. Anal. 188 (2008), 457-473.

[16] R. Coifman, P.-L. Lions, Y. Meyer, and S. Semmes, Compensated compactness and Hardy spaces, J. Math. Pures Appl. 72 (1993), 247-286.

[17] S. Conti, C. De Lellis, and L. Székelyhidi Jr., h-principle and rigidity for $C^{1, \alpha}$ isometric embeddings, In: Nonlinear Partial Differential Equations, pp. 83-116, H. Holden and K. Karlsen (Eds.), Springer-Verlag: Berlin-Heidelberg, 2012.

[18] S. Conti, G. Dolzmann, and S. Müller, The div-curl lemma for sequences whose divergence and curl are compact in $W^{-1,1}$, C. R. Math. Acad. Sci. Paris, 349 (2011), 175-178.

[19] M. P. do Carmo, Riemannian Geometry, Birkhäuser: Boston, 1992.

[20] B. Dacorogna, Weak Continuity and Weak Lower Semicontinuity of Nonlinear Functionals, Springer-Verlag: Berlin, 1982.

[21] C. M. Dafermos, Hyperbolic Conservation Laws in Continuum Physics, Third Ed., Springer-Verlag: Berlin, 2010.

[22] L. P. Eisenhart, Riemannian Geometry, Eighth Printing, Princeton University Press: Princeton, NJ, 1997.

[23] L. C. Evans, Weak Convergence Methods for Nonlinear Partial Differential Equations, CBMS-RCSM, 74, AMS: Providence, 1990.

[24] L. C. Evans and S. Müller, Hardy spaces and the two-dimensional Euler equations with nonnegative vorticity, J. Amer. Math. Soc. 7 (1994), 199-219. 
[25] M. Fabian, P. Habala, P. Hájek, V. M. Santalucía, J. Pelant, and V. Zizler, Functional Analysis and Infinite-Dimensional Geometry, Springer: New York, 2001.

[26] H. F. Goenner, On the interdependency of the Gauss-Codazzi-Ricci equations of local isometric embedding, General Relativity and Gravitation, 8 (1977), 139145 .

[27] R. Greene, Isometric Embeddings of Riemannian and Pseudo-Riemannian Manifolds, Mem. Amer. Math. Soc. 97, AMS: Providence, RI, 1970.

[28] M. Gromov, Partial Differential Relations, Vol. 9. Springer Science and Business Media, 2013.

[29] M. Günther, Zum Einbettungssatz von J. Nash, Math. Nach. 144 (1989), 165187.

[30] Q. Han and J.-X. Hong, Isometric Embedding of Riemannian Manifolds in Euclidean Spaces, AMS: Providence, 2006.

[31] T. Iwaniec, A. Lutoborski, Integral estimates for null Lagrangians, Arch. Rational Mech. Anal., 125 (1993), 25-79.

[32] M. Janet, Sur la possibilité de plonger un espace Riemannian donné dans un espace Euclidien, Ann. Soc. Pol. Math. 5 (1926), 38-43.

[33] H. Kozono and T. Yanagisawa, Global compensated compactness theorem for general differential operators of first order, Arch. Rational Mech. Anal. 207 (2013), 879-905.

[34] J. Langer, A compactness theorem for surfaces with $L_{p}$-bounded second fundamental form, Math. Ann. 270 (1985), 223-234.

[35] P.G. LeFloch, C. Mardare, and S. Mardare, Isometric immersions into the Minkowski spacetime for Lorentzian manifolds with limited regularity, Disc. Cont. Dyn. Syst. 23 (2009), 341-365.

[36] S. Mardare, On Pfaff systems with $L^{p}$ coefficients and their applications in differential geometry, J. Math. Pures Appl. 84 (2005), 1659-1692.

[37] S. Mardare, On systems of first order linear partial differential equations with $L^{p}$ coefficients, Adv. Diff. Eq. 12 (2007), 301-360.

[38] M. Mars, J. M. M. Senovilla, Geometry of general hypersurfaces in spacetime: junction conditions, Class. Quantum Grav. 10 (1993), 1865-1897.

[39] F. Murat, Compacité par compensation, Ann. Scuola Norm. Sup. Pisa Cl. Sci. 5 (1978), 489-507.

[40] F. Murat, Compacité par compensation. II, In: Proceedings of the International Meeting on Recent Methods in Nonlinear Analysis (Rome, 1978), pp. 245-256, Pitagora, Bologna, 1979.

[41] G. Nakamura and Y. Maeda, Local isometric embedding problem of Riemannian 3-manifold into $\mathbb{R}^{6}$, Proc. Japan Acad. Ser. A: Math. Sci. 62 (1986), 257-259.

[42] J. Nash, $C^{1}$ isometric imbeddings, Ann. Math. 60 (1954), 383-396.

[43] J. Nash, The imbedding problem for Riemannian manifolds, Ann. Math. 63 (1956), 20-63. 
[44] B. O'Neill, Semi-Riemannian Geometry with Applications to Relativity, Vol. 103. Academic Press, 1983.

[45] J. Robbin, R. Rogers, and B. Temple, On weak continuity and the Hodge decomposition, Trans. Amer. Math. Soc. 303 (1987), 609-618.

[46] M. Spivak, A Comprehensive Introduction to Differential Geometry, Publish or Perish, Inc.: Boston, Mass., Vol. I-II, 1970; Vol. III-V, 1975.

[47] S. Sternberg, Curvature in Mathematics and Physics, Courier Corporation, 2012.

[48] J. A. Schouten, Ricci-Calculus: An Introduction to Tensor Analysis and Its Geometrical Applications, Vol. 10. Springer Science and Business Media, 2013.

[49] M. Szopos, An existence and uniqueness result for isometric immersions with little regularity, Rev. Roumaine. Math. Pures Appl. 53 (2008), 555-565.

[50] L. Tartar, Compensated compactness and applications to partial differential equations, In: Nonlinear Analysis and Mechanics: Heriot-Watt Symposium, Vol. 4, pp. 136-212, Res. Notes in Math. 39, Pitman: Boston, Mass.-London, 1979.

[51] L. Tartar, The compensated compactness method applied to systems of conservation laws. In: Systems of Nonlinear Partial Differential Equations (Oxford, 1982), pp. 263-285, NATO Adv. Sci. Inst. Ser. C Math. Phys. Sci., 111, Reidel: Dordrecht, 1983.

[52] K. Tenenblat, On isometric immersions of Riemannian manifolds, Bull. Brazilian Math. Soc. 2 (1971), 23-36.

[53] F. W. Warner, Foundations of Differentiable Manifolds and Lie Groups, Scott, Foresman and Co.: Glenview, Ill.-London, 1971.

[54] H. Whitney, Geometric Integration Theory, Princeton University Press: Princeton, 1957.

[55] S.-T. Yau: Review of geometry and analysis. In: Mathematics: Frontiers and Perspectives, pp. 353-401. International Mathematics Union, Eds. V. Arnold, M. Atiyah, P. Lax, B. Mazur, AMS: Providence, RI, 2000.

Mathematical Institute, University of Oxford, Oxford, OX2 6GG, UK

E-mail: chengq@maths.ox.ac.uk

Mathematical Institute, University of Oxford, Oxford, OX2 6GG, UK

E-mail: Siran.Li@rice.edu 